\title{
Gracefullness of Building Graph of Level Five
}

\author{
A.Solairaju \\ Associate Professor \& Research Advisor, \\ PG \& Research Department of Mathematics, \\ Jamal Mohamed College Tiruchirappalli, \\ Tamilnadu, India
}

\author{
T. Narppasalaiarasu \\ Assistant Professor of Mathematics, \\ Chikkanna Government Arts College, \\ Tiruppur
}

\begin{abstract}
In this article, the gracefulness of the building graph of level five is obtained.
\end{abstract}

\section{KeyWords}

Path, circuit, graph labeling, Graceful graphs, graceful numbering

\section{INTRODUCTION}

Rengel [1964] conjecture mentions that the complete graph $\mathrm{K} 2 \mathrm{n}+1$ can be decomposed into $2 \mathrm{n}+1$ copies of a given tree with $n$ edges. Rosa [4] introduced graceful labeling as a tool to solve the Rengel's conjecture. A function $\mathrm{f}$ is called a graceful labeling of a graph with $\mathrm{m}$ edges, if $\mathrm{f}$ is an injection from the vertex set of $G$ to the set $\{0,1,2, \ldots, m\}$ such that when each edge uv is assigned the label $|f(u)-f(v)|$ the resulting edge labels are distinct.

Rosa introduced graceful graphs and graceful labeling methods in 1967. The graceful labeling problem is to determine which graphs are graceful. Golomb [1972] further studied such labeling. After 1972, several hundreds papers have been published on graceful labeling methods. Most of the papers are theoretical. While the graceful labeling of graphs is assumed to be a theoretical subject, these graphs serve as models in many areas like coding theory, communication network, etc. A complete summary of graceful and non-graceful graphs and the results along with some unproven conjectures can be found in Gallian's dynamic survey of graceful labeling [2008]. The survey reveals that the gracefulness of several classes of graphs has already been established. For example, all the paths Pn, all the trees, the complete graphs $\mathrm{Kn}$ for $\mathrm{n}<5$, all the wheels, etc., are graceful.

Solairaju et.al. [2008, 2009] proved few results towards evenedge gracefulness of trees.

\section{THE GRACEFULNESS OF THE BUILDING GRAPH OF LEVEL FIVE}

\section{Definition 2.1}

Graceful Graph: A function $\mathrm{f}$ of a graph $\mathrm{G}$ is called a graceful labeling with $\mathrm{m}$ edges, if $\mathrm{f}$ is an injection from the vertex set of $G$ to the set $\{0,1,2, \ldots, m\}$ such that when each edge uv is assigned the label $|\mathrm{f}(\mathrm{u})-\mathrm{f}(\mathrm{v})|$ the resulting edge labels are distinct. Hence the graph $\mathrm{G}$ is graceful.

\section{Definition 2.2}

Path: An open walk in which no vertex appears more than once is called a path. We denote Pn as a path with ' $n$ ' vertices where $\mathrm{n} \geq 1$.

\section{Definition 2.3}

Circuit: A closed walk in which no vertex (except the initial and the final vertex) appears more than once is called a circuit. We denote $\mathrm{Cn}$ as a circuit with ' $\mathrm{n}$ ' vertices where $\mathrm{n} \geq$ 1.

\section{Definition 2.4}

The Building graph of level five is a connected graph $B(n)$ defined as in figure 1 . Note that the graph contains 5 vertices from top to down, and a finite number of copies like this.

Main theorem 2.5: The Building graph of level five is graceful graph.

\section{Proof}

Some arbitrary labeling for the vertex set of given building graph is as follows:
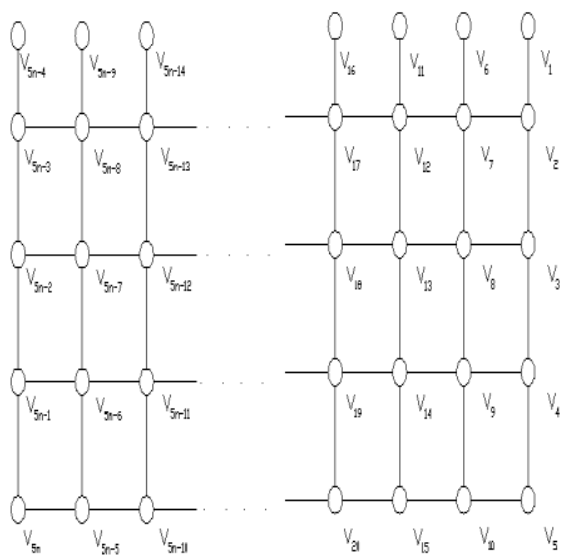

Figure 1 - Building graph of level five

Define a map $\mathrm{f}: \mathrm{v}(\mathrm{G}) \rightarrow\{0, \ldots \mathrm{q}\}$ by

Rule 1

$$
\begin{aligned}
& f\left(v_{i}\right)=\frac{i-1}{2}, i=1,3,5 \\
& f\left(v_{2}\right)=q \text { and } f\left(v_{4}\right)=(q-1) .
\end{aligned}
$$

Rule 2

$$
\begin{aligned}
& f\left(v_{i}\right)=q-\left(\frac{i}{2}\right), i=6,8,10 \quad \text { and } \\
& f\left(v_{i}\right)=\frac{i+1}{2}, i=7,9
\end{aligned}
$$

Rule 3
(i) $\mathrm{n}$ is odd and $\mathrm{j}=11,21,31, \ldots, .(5 \mathrm{n}-4)$
(or) $\mathrm{n}$ is even and $\mathrm{j}=11,21,31, \ldots,(5 n-9)$.

$$
f\left(v_{j}\right)=f\left(v_{1}\right)+\left[\frac{j}{10}\right] 7
$$
(ii) $n$ is odd and $\mathrm{j}=16,26,36, \ldots .,(5 n-9)$ (or) 
$n$ is even and $j=16,26,36, \ldots,(5 n-4)$.

$$
f\left(v_{j}\right)=f\left(v_{6}\right)-\left[\frac{j}{10}\right] 7
$$

\section{Rule 4}

(i) $n$ is odd and $j=12,22,32, \ldots,(5 n-3)$ (or)

$n$ is even and $j=12,22,32, \ldots,(5 n-8)$.

$f\left(v_{j}\right)=f\left(v_{2}\right)-\left[\frac{j}{10}\right] 7$

(ii) $n$ is odd and $\mathrm{j}=17,27,37, \ldots,(5 n-8)$

(or) $n$ is even and $j=17,27,37, \ldots,(5 n-3) . f\left(v_{j}\right)=f\left(v_{7}\right)+$ $\left[\frac{\mathrm{j}}{10}\right] 7$

\section{Rule 5}

(i) $n$ is odd and $\mathrm{j}=13,23,33, \ldots,(5 \mathrm{n}-2)$ (or)

$n$ is even and $j=13,23,33, \ldots,(5 n-7)$.

$$
f\left(v_{j}\right)=f\left(v_{3}\right)+\left[\frac{j}{10}\right] 7
$$

(ii) $n$ is odd and $j=18,28,38, \ldots,(5 n-7)$ (or)

$\mathrm{n}$ is even and $\mathrm{j}=18,28,38, \ldots, .(5 n-2)$.

$$
f\left(v_{j}\right)=f\left(v_{8}\right)-\left[\frac{j}{10}\right] 7
$$

\section{Rule 6}

(i) $n$ is odd and $j=14,24,34, \ldots$, (5n-1) (or) $n$ is even and $j=14,24,34, \ldots .,(5 n-6)$.

$$
f\left(v_{j}\right)=f\left(v_{4}\right)-\left[\frac{j}{10}\right] 7
$$

(ii) $n$ is odd and $j=19,29,39, \ldots .,(5 n-6)$ (or)

$\mathrm{n}$ is even and $\mathrm{j}=19,29,39, \ldots ., .(5 \mathrm{n}-1)$.

$f\left(v_{j}\right)=f\left(v_{9}\right)+\left[\frac{j}{10}\right] 7$

Rule 7

(i) $n$ is odd and $j=15,25,35, \ldots$, (5n) (or)

is even and $j=15,25,35, \ldots .,(5 n-5)$.

$$
\mathrm{f}\left(\mathrm{v}_{\mathrm{j}}\right)=\mathrm{f}\left(\mathrm{v}_{5}\right)+\left[\frac{\mathrm{j}}{10}\right] 7
$$

(ii) $n$ is odd and $\mathrm{j}=20,30,40, \ldots,(5 n-5)$ (or)

$n$ is even and $j=20,30,40, \ldots, .(5 n)$.

$$
f\left(v_{j}\right)=f\left(v_{10}\right)-\left[\frac{j}{10}\right] 7
$$

\section{Example 2.6}

A building graph $\mathrm{B}(11)$ is graceful as in the figure 2 mentioned below.

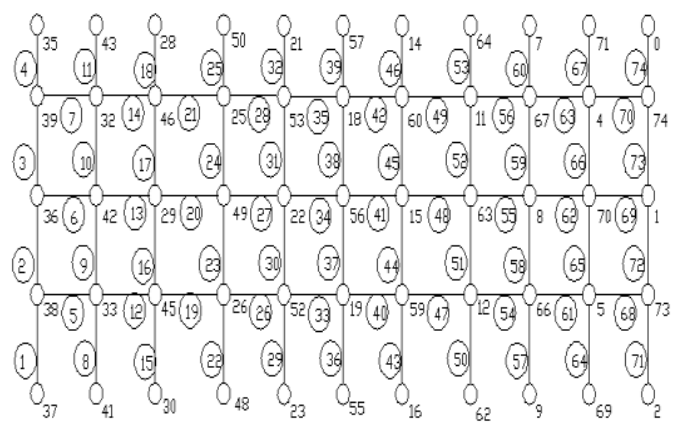

Example 2.7: A building graph $\mathrm{B}(10)$ is graceful graph as in the figure 2 mentioned below.

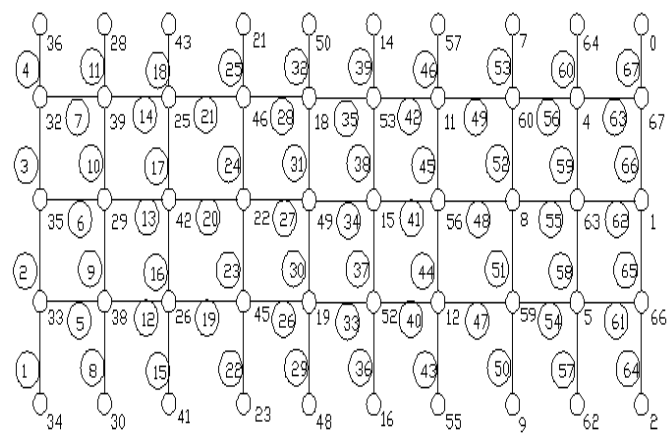

\section{REFERENCES}

[1] F. Harary, Graph Theory, Addison. Wesley, Reading. Mass (1969)

[2] J.A.Gallian, A dynamic survey of graph labeling, Electro.J.Combin. 15 (2008) \#DS6.

[3] G.Ringel , Problem 25, in Theory of Graphs and its Applications, Proc. Symposium Smolenice 1963, Prague (1964) 162.

[4] A Rosa, On certain valuations of the vertices of a graph, Theory of Graphs (International Symposium, Rome, July 1966), Gordon and Breach, N.Y. and Dunod Paris (1967) $349-355$.

[5] S.W Golomb, How to number a graph, in Graph Theory and computing, R.C. Read, ed. Academic press, New York (1972) $23-27$

[6] A.Solairaju, A.Sasikala, C.Vimala Gracefulness of a spanning tree of the graph of product of $\mathrm{Pm}$ and $\mathrm{Cn}$, The Global Journal of Pure and Applied Mathematics of Mathematical Sciences, Vol.1, No-2 (July-Dec 2008): 133-136

[7] A.Solairaju and K.Chitra Edge-odd graceful labeling of some graphs " Electronics Notes in Discrete Mathematics Volume 33,April 2009, Pages 15 - 20

[8] A.Solairaju, C.Vimala, A.Sasikala Gracefulness of a spanning tree of the graph of Cartesian product of Sm and $\mathrm{Sn}$, The Global Journal of Pure and Applied Mathematics of Mathematical Sciences, Vol. 1, No-2 (July-Dec 2008): 117-120

[9] A.Solairaju, A.Sasikala, C.Vimala Edge-odd Gracefulness

ofaspanningtreeofCartesianproductofP2andCninPacificsl of Mathematics, Vol .3, No. 1-2. Jan-Dec. 2009, :39-42)

[10] A.Solairaju, C.Vimala, A.Sasikala Even Edge Gracefulness of the Graphs in Pacific-Asian Journal of Mathematics, Vol. 3, No. 1-2. Jan-Dec. 2009, :43-47) 